\title{
An application of canonical variate analysis in profile comparison of dry matter content of white clover between ozone-sensitive and ozone-resistant clones exposed in ambient air conditions
}

\author{
Dariusz Kayzer ${ }^{1}$, Anna Budka ${ }^{1}$, Klaudia Borowiak ${ }^{2}$, Janina Zbierska ${ }^{2}$, \\ Marta Lisiak ${ }^{2}$ \\ ${ }^{1}$ Department of Mathematical and Statistical Methods, Poznan University of Life Sciences, \\ Wojska Polskiego 28, 60-637 Poznań, Poland, e-mail: dkayzer@up.poznan.pl; \\ budka@up.poznan.pl \\ ${ }^{2}$ Department of Ecology and Environmental Protection, Poznan University of Life Sciences, \\ Piątkowska 94C, 60-649 Poznań, Poland, e-mail: klaudine@up.poznan.pl; jzbier@up.poznan.pl; \\ lismar@up.poznan.pl
}

\section{SUMMARY}

\begin{abstract}
Tropospheric ozone affects plant growth and the yield of main pasture species all around the world. Experiments are usually performed in fully controlled conditions; the number of investigations in ambient air conditions is still limited. Moreover, most investigations of the effect of ozone on white clover biomass production consider one series after the other, including a period without leaves. Hence, based on the recommendations, additional series are proposed and studied here. The responses of sensitive and resistant white clover clones are presented and compared using multivariate analysis of variance and profile analysis. The canonical variate analysis used here makes it possible to present the profile comparison of dry matter content of white clover graphically in Euclidean space. The investigations revealed a difference in response between clones and the necessity of using the additional series.
\end{abstract}

Key words: multivariate analysis of variance, profile analysis, canonical variate analysis, ozone, dry weight, white clover

\section{Introduction}

Bioindication methods can be a supplement to automatic monitoring systems of air pollution, based on the response of living organisms to air pollution. This is especially important for oxidative pollutants, such as ozone or nitrogen oxides, 
because they do not accumulate in organisms. Hence visible injury caused by air pollution can be a good indirect indicator of their negative effect on living organisms. Moreover, plants have also revealed other symptoms of negative tropospheric ozone impact, such as lower level of photosynthesis activity and decreased productivity, and furthermore a decrease in biomass production and yield losses can also be noted (Rai et al. 2007, Feng et al. 2008, Vandermeiren et al. 2005, Shi et al. 2009, Hassan and Tewfik 2006). The last can be an important cause of economic losses (van Dingenen et al. 2009). Hence it is important to assess the potential effect of air pollutants on these parameters. Some plant species are well known as bioindicators for ozone, such as tobacco and its two cultivars (Borowiak et al. 2012, Klumpp et al. 2006), while others are still being examined. White clover (Trifolium repens L. cv. 'Regal') was investigated during the ICP Vegetation programme, according to the Geneva Convention on Long-Range Trans-boundary Air Pollution, for several years to assess the negative effect of tropospheric ozone on vegetation. Two clones ozone-sensitive and ozone-resistant - were examined far from local air pollution sources to evaluate the effect of ozone on plants with regard to far-source pollution (Fumagilli et al. 2003). Tropospheric ozone was found to be one of the most harmful air pollutants to crops, because its highest concentrations occur during the growing season (Elagöz and Manning 2005), and mostly in remote areas. Hence it is very important to investigate the effect of this pollutant on vegetation and its production.

The aim of the study was to evaluate the effect of tropospheric ozone on dry matter content of white clover exposed at remote areas for four-week periods. Plant response was compared during various series and between white clover clones. The study also served to evaluate the usefulness of canonical variate analysis in obtaining answers to the above-mentioned questions. 


\section{Materials and methods}

\subsection{Experimental design}

Ozone-sensitive (NC-S) and ozone-resistant (NC-R) clones of white clover ( $T$. repens) were used in the experiment. Seedlings of both clones were obtained from the chief coordinator of the ICP Vegetation programme. Plants were exposed to ozone under natural conditions in a place located about $20 \mathrm{~km}$ from Poznan city; the location was chosen according to the methodology of that programme (Fumagilli et al. 2003, ICP Vegetation 2003). Ten exposure series were carried out in the growing season of 2005. During the experiment the same plants, earlier cultivated in greenhouse conditions for four weeks, were exposed to ambient air. Each exposure series lasted four weeks, after which the dry mass of the above-ground parts of the plants was analysed. For this purpose the whole above-ground part of plants - leaves, stems and flowers - was cut and dried. To avoid a time between series without plants due to the cutting of the previous series, the second series of plants (series B) overlapped series A by two weeks (Figure 1).

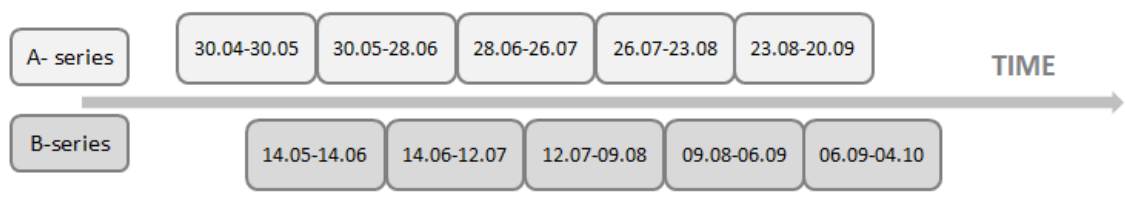

Figure 1. Dates of white clover exposure series

Plants were exposed to field conditions in the Tarnowo Podgórne area, about 15 $\mathrm{km}$ west of the city of Poznan, Poland. As recommended by the ICP Vegetation programme, the exposure site was located at least $200 \mathrm{~m}$ from main roads and $50 \mathrm{~m}$ from buildings. Each series consisted of 30 plants ( 15 of each clone). Dry weight content was measured after every four-week exposure. 


\subsection{Mathematical model of observation}

Assume that the field given by the $h$-th white clover harvest in the $j$-th series for the $i$-th clone in the $k$-th replication will be assigned by:

$$
y_{i j k}(h)=\mu(h)+\alpha_{i}(h)+\beta_{j}(h)+\gamma_{i j}(h)+e_{i j k}(h)
$$

where $\mu(h)$ represents the general level of dry weight content in the $h$-th harvest, $\alpha_{i}(h)$ is the effect of the $i$-th clone from the $h$-th harvest, $\beta_{j}(h)$ is the effect of the $j$-th series from the $h$-th harvest, $\gamma_{i j}(h)$ is the interaction effect of clone $i$ with series $j$ and the $h$-th harvest, $e_{i j k}(h)$ is the error related to the $i, j, k$, $h$-th combination of clone, series, replications and harvest. We assume that random errors for each harvest have independent normal distribution with zero expected value and with variance $\sigma_{e}^{2}(h)$.

The indices presented in this experiment take the following values: $i=1,2$; $j=1,2, k=1, . ., 15, h=1, . ., H(H=5)$. Hence, we obtained $N=2 \cdot 2 \cdot 15=60$ plants analysed in the experiment. Due to correlations between the dry weight content of certain harvests, it is sufficient to use tools of multivariate analysis of variance for determination the differences between series and clones. Hence, the considered model (1) for one plant of white clover can be presented in the form:

$$
\mathbf{y}_{i j k}=\boldsymbol{\mu}+\boldsymbol{\alpha}_{i}+\boldsymbol{\beta}_{j}+\boldsymbol{\gamma}_{i j}+\mathbf{u}_{i j k}
$$

where $\quad \mathbf{y}_{i j k}=\left[y_{i j k}(1), \ldots, y_{i j k}(H)\right]^{\prime}, \quad \boldsymbol{\mu}=[\mu(1), \ldots, \mu(H)]^{\prime}, \quad \boldsymbol{\alpha}_{i}=\left[\alpha_{i}(1), \ldots, \alpha_{i}(H)\right]^{\prime}$, $\boldsymbol{\beta}_{j}=\left[\beta_{j}(1), \ldots, \beta_{j}(H)\right]^{\prime}, \quad \gamma_{i j}=\left[\gamma_{i j}(1), \ldots, \gamma_{i j}(H)\right]^{\prime}, \quad \mathbf{u}_{i j k}=\left[e_{i j k}(1), \ldots, e_{i j k}(H)\right]^{\prime}$. Finally the model (2) can be described in the matrix form

$$
\mathbf{Y}=\mathbf{1}_{N} \boldsymbol{\mu}^{\prime}+\mathbf{X}_{1} \boldsymbol{\alpha}+\mathbf{X}_{2} \boldsymbol{\beta}+\mathbf{X}_{12} \boldsymbol{\gamma}+\mathbf{U}
$$

where: $\quad \mathbf{Y}=\left[\mathbf{y}_{1,1,1}, \ldots, \mathbf{y}_{1,1,15}, \mathbf{y}_{1,2,1}, \ldots, \mathbf{y}_{2,2,1}\right]^{\prime}, \quad \boldsymbol{\alpha}=\left[\boldsymbol{\alpha}_{1}, \boldsymbol{\alpha}_{2}\right]^{\prime}, \quad \boldsymbol{\beta}=\left[\boldsymbol{\beta}_{1}, \boldsymbol{\beta}_{2}\right]^{\prime}$, $\boldsymbol{\gamma}=\left[\gamma_{1,1}, \gamma_{1,2}, \gamma_{2,1}, \gamma_{2,2}\right]^{\prime}, \mathbf{U}=\left[\mathbf{u}_{1,1,1}, \ldots, \mathbf{u}_{1,1,15}, \mathbf{u}_{1,2,1}, \ldots, \mathbf{u}_{2,2,15}\right]^{\prime}, \mathbf{X}_{1}=\mathbf{I}_{2} \otimes \mathbf{1}_{2} \otimes \mathbf{1}_{15}$, $\mathbf{X}_{2}=\mathbf{1}_{2} \otimes \mathbf{I}_{2} \otimes \mathbf{1}_{15}, \quad \mathbf{X}_{12}=\mathbf{I}_{2} \otimes \mathbf{I}_{2} \otimes \mathbf{1}_{15}$ (the symbol $\otimes$ denotes the Kronecker product of matrices). 


\subsection{Statistical hypotheses in two-way multivariate analysis of variance}

In the presented experiment the hypothesis of absence of differences between the average yields of dry weight of the Sensitive and Resistant clones is presented in the form:

$$
\mathrm{H}_{01}: \mathbf{C}_{1}^{\prime} \boldsymbol{\alpha}=\mathbf{0},
$$

where the vector $\mathbf{C}_{1}$ can be written in the form: $\mathbf{C}_{1}^{\prime}=\left[\begin{array}{ll}1 & -1\end{array}\right]$.

The hypothesis of absence of differences between the average yields of dry matter in Series A and Series B is presented in the form:

$$
\mathrm{H}_{02}: \mathbf{C}_{2}^{\prime} \boldsymbol{\beta}=\mathbf{0},
$$

where vector $\mathbf{C}_{2}$ can be written in the form: $\mathbf{C}_{2}^{\prime}=\left[\begin{array}{ll}1 & -1\end{array}\right]$.

The hypothesis of absence of clone-series interaction is presented in the form:

$$
\mathrm{H}_{012}: \mathbf{C}_{12} \boldsymbol{\gamma}=\mathbf{0}
$$

where matrix $\mathbf{C}_{12}$ can be written in the form: $\mathbf{C}_{12}=\left(\mathbf{I}_{2}-\frac{1}{2} \mathbf{1}_{2} \mathbf{1}_{2}^{\prime}\right) \otimes\left(\mathbf{I}_{2}-\frac{1}{2} \mathbf{1}_{2} \mathbf{1}_{2}^{\prime}\right)$. The hypotheses (3-5) were tested using Lawley-Hotelling statistics $(s=1,2,12)$ in the form:

$$
T_{0 s}^{2}=\operatorname{trace}\left(\mathbf{S}^{-1} \mathbf{H}_{s}\right),
$$

where:

$\mathbf{H}_{1}=\left(\mathbf{C}_{1}^{\prime} \hat{\boldsymbol{\alpha}}\right)^{\prime}\left[\mathbf{C}_{1}^{\prime}\left(\mathbf{X}_{1}^{\prime} \mathbf{X}_{1}\right)^{-1} \mathbf{C}_{1}\right]^{-}\left(\mathbf{C}_{1}^{\prime} \boldsymbol{\alpha}\right), \quad \mathbf{H}_{2}=\left(\mathbf{C}_{2}^{\prime} \hat{\boldsymbol{\beta}}\right)^{\prime}\left[\mathbf{C}_{2}^{\prime}\left(\mathbf{X}_{2}^{\prime} \mathbf{X}_{2}\right)^{-1} \mathbf{C}_{2}\right]^{-}\left(\mathbf{C}_{2}^{\prime} \hat{\boldsymbol{\beta}}\right) \quad$ and $\mathbf{H}_{12}=\left(\mathbf{C}_{12} \hat{\gamma}\right)^{\prime}\left[\mathbf{C}_{12}\left(\mathbf{X}_{12}^{\prime} \mathbf{X}_{12}\right)^{-1} \mathbf{C}_{12}^{\prime}\right]^{-}\left(\mathbf{C}_{12} \boldsymbol{\gamma}\right), \quad \mathbf{S}=(N-r)^{-1} \mathbf{Y}^{\prime}\left(\mathbf{I}_{N}-\mathbf{X}\left(\mathbf{X}^{\prime} \mathbf{X}\right)^{-} \mathbf{X}^{\prime}\right) \mathbf{Y}$ and $\mathbf{X}=\left[\mathbf{1}_{N} \vdots \mathbf{X}_{1} \vdots \mathbf{X}_{2} \vdots \mathbf{X}_{12}\right]$ is a matrix of rank $r$. In addition, the best linear unbiased estimator for $\boldsymbol{\alpha}, \boldsymbol{\beta}, \boldsymbol{\gamma}$ can be presented in the form $\hat{\boldsymbol{\alpha}}=\left(\mathbf{X}_{1}^{\prime} \mathbf{X}_{1}\right)^{-1} \mathbf{X}_{1}^{\prime} \mathbf{Y}$, $\hat{\boldsymbol{\beta}}=\left(\mathbf{X}_{2}^{\prime} \mathbf{X}_{2}\right)^{-1} \mathbf{X}_{2}^{\prime} \mathbf{Y}$ and $\hat{\boldsymbol{\gamma}}=\left(\mathbf{X}_{12}^{\prime} \mathbf{X}_{12}\right)^{-1} \mathbf{X}_{12}^{\prime} \mathbf{Y}$ (Anderson 2003, Morrison 1967, Seber 1980, 1984).

Using approximation (McKeon 1974), the hypothesis $\mathrm{H}_{0 s}$ was rejected, when $F_{s}=T_{0 s}^{2} /(N-r) c_{s}>F_{a_{s}, b_{s}}(\alpha)$, where $c_{s}=a_{s}\left(b_{s}-2\right) /\left[b_{s}(N-r-H-1)\right]$, $a_{s}=H \cdot \operatorname{rank} \mathbf{C}_{s}, b_{s}=4+\left(a_{s}+2\right) /\left(B_{s}-1\right)$ with 


$$
B_{s}=\frac{\left(N-r+\operatorname{rank} \mathbf{C}_{s}-H-1\right)(N-r-1)}{(N-r-H-3)(N-r-H)}
$$

(Lejeune and Caliński 2000).

\subsection{Statistical hypotheses in profile analysis}

Another way to test the hypotheses $\mathrm{H}_{01}, \mathrm{H}_{02}, \mathrm{H}_{012}$ is to consider the hypothesis, of interest from the point of view of the experimenter:

$$
\mathrm{H}_{03}: \mathbf{C}_{3} \boldsymbol{\gamma}=\mathbf{0} \text {, }
$$

where $\mathbf{C}_{3}=\mathbf{I}_{4}-\frac{1}{4} \mathbf{1}_{4} \mathbf{1}_{4}^{\prime}$. Using the matrix $\mathbf{C}_{3}$ the differences between experimental objects are analysed. In this case it is defined as dry weight content for a certain clone exposed in certain exposure series. An object effect is determined as differences between values of dry weight content for a particular experimental object and means for all objects. Note, however, that the matrix $\mathbf{S}$ may be calculated from the form $\mathbf{S}=(N-4)^{-1} \mathbf{Y}^{\prime}\left(\mathbf{I}_{N}-\mathbf{X}_{12}\left(\mathbf{X}_{12}^{\prime} \mathbf{X}_{12}\right)^{-1} \mathbf{X}_{12}^{\prime}\right) \mathbf{Y}$. If the hypothesis $\mathrm{H}_{03}$ is rejected, this may be either due to the fact the clone factor causes some interaction with the series factor, or because there are some main effects of the former factor, or both.

The profile analysis can also verify the hypothesis of no differences for any combination between clones and series in terms of dry weight content. These hypotheses can be written in the following form:

$$
\mathrm{H}_{0 t}: \mathbf{c}_{t}^{\prime} \boldsymbol{\gamma}=0
$$

where the 4 - dimensional vector $\mathbf{c}_{t}$ describes the contrast issue (Caliński et al. 1975). Hypotheses $\mathrm{H}_{03}$ using the statistic $T_{0 t}^{2}=\left[\mathbf{c}_{t}^{\prime}\left(\mathbf{X}_{12}^{\prime} \mathbf{X}_{12}\right)^{-} \mathbf{c}_{t}\right]^{-1} \mathbf{c}_{t}^{\prime} \hat{\gamma} \mathbf{S}^{-1} \hat{\gamma}^{\prime} \mathbf{c}_{t}$ are tested.

The test statistic $F_{t}=(N-r-H+1) T_{0 t}^{2} /((N-r) H)$ denotes that the statistic has $F$ distribution with $H$ and $(N-r-H+1)$ degrees of freedom when the null

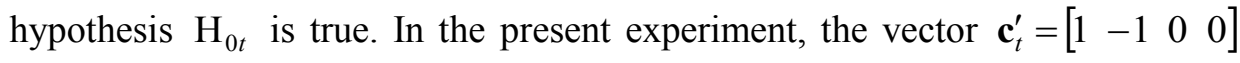
denotes that we are testing whether differences between mean dry weight 
contents of the sensitive clone vary between series A and B for certain harvests. Moreover, the statistics $T_{0 t h}^{2}=\left[\mathbf{c}_{t}^{\prime}\left(\mathbf{X}_{12}^{\prime} \mathbf{X}_{12}\right) \mathbf{c}_{t}\right]^{-1}\left(\mathbf{c}_{t}^{\prime} \hat{\gamma} \mathbf{d}_{h}\right)^{2} / S_{h}$ where $S_{h}$ is the $h$-th diagonal element of matrix $\mathbf{S}$ and $\mathbf{d}_{h}$ is the $h$-th column of $\mathbf{I}_{H}$ matrix ( $T_{0 t h}^{2}$ has a Student distribution with $N-r$ degrees of freedom) tests which harvests in the examined contrast have an influence on the rejection of hypothesis $\mathrm{H}_{0 t}$. A similar treatment can be applied to the hypotheses

$$
\mathrm{H}_{0 t}: \mathbf{c}_{t}^{\prime} \gamma \mathbf{1}_{H}=0
$$

which test differences between individual experimental objects, summing over all harvests.

\subsection{Canonical variate analysis}

For the graphical presentation of clone-series interaction, canonical variate analysis was used. This is based on transforming the matrix $\mathbf{C}_{3} \hat{\gamma}$ into a set of new variables, which carry similar information, but are distributed in a multivariate Euclidean space (Lejeune and Caliński 2000, Kayzer et al. 2009, Borowiak et al. 2011, Budka et al. 2011). Following the transformation, the matrix $\mathbf{C}_{3} \hat{\gamma}$ is presented in the form: $\mathbf{C}_{3} \hat{\gamma}=\sum_{w=1}^{v} \lambda_{w}^{-1 / 2} \boldsymbol{\Psi}_{w} \boldsymbol{\varphi}_{w}^{\prime}$, where $v=\min (H, 3)$ and the vectors $\boldsymbol{\psi}_{w}$ and $\boldsymbol{\varphi}_{w}$ and scalars $\lambda_{w}$ are determined from equations of the form:

$$
\begin{aligned}
& \mathbf{C}_{3} \hat{\gamma} \mathbf{S}^{-1}\left(\mathbf{C}_{3} \hat{\gamma}\right)^{\prime}\left[\mathbf{C}_{3}\left(\mathbf{X}_{12} \mathbf{X}_{12}^{\prime}\right)^{-1} \mathbf{C}_{3}^{\prime}\right]^{-} \boldsymbol{\Psi}_{w}=\lambda_{w} \boldsymbol{\Psi}_{w}, \\
& \left(\mathbf{C}_{3} \hat{\gamma}\right)^{\prime}\left[\mathbf{C}_{3}\left(\mathbf{X}_{12}^{\prime} \mathbf{X}_{12}\right)^{-1} \mathbf{C}_{3}^{\prime}\right]^{-}\left(\mathbf{C}_{3} \hat{\gamma}\right) \mathbf{S}^{-1} \boldsymbol{\varphi}_{w}=\lambda_{w} \boldsymbol{\varphi}_{w} .
\end{aligned}
$$

The vectors $\psi_{w}$ and $\varphi_{w}$ are standardized in the following way:

$$
\begin{aligned}
& \boldsymbol{\Psi}_{w}^{\prime}\left[\mathbf{C}_{3}\left(\mathbf{X}_{12}^{\prime} \mathbf{X}_{12}\right)^{-1} \mathbf{C}_{3}^{\prime}\right]^{-} \boldsymbol{\Psi}_{w^{\prime}}=\left\{\begin{array}{lll}
\lambda_{w}, & \text { if } & w=w^{\prime} \\
0, & \text { if } & w \neq w^{\prime}
\end{array}\right. \\
& \boldsymbol{\varphi}_{w}^{\prime} \mathbf{S}^{-1} \boldsymbol{\varphi}_{w^{\prime}}= \begin{cases}\lambda_{w}, & \text { if } w=w^{\prime} \\
0, & \text { if } w \neq w^{\prime}\end{cases}
\end{aligned}
$$




\section{Results and discussion}

The effects of the various sources of variation were examined using multivariate analysis of variance. The results of the testing of the hypotheses, according to the formula (6), are presented in Table 1. It can be concluded that the dry weight content varies due to clones, series and interaction between clone and series, because the respective empirical significance levels are lower than 0.05 .

Table 1. Results of tests conducted in profile analysis for investigation of the structure of dry weight content

\begin{tabular}{cccc}
\hline Source of variability & Test statistics $T_{0 s}^{2}$ & Test statistics $F_{s}$ & $\begin{array}{c}\text { Empirical } \\
\text { significance } \\
\text { level } p\end{array}$ \\
\hline clones & 33.6 & 6.25 & $<0.01$ \\
series & 339.2 & 63.2 & $<0.01$ \\
interaction & 18.9 & 3.51 & 0.01 \\
\hline
\end{tabular}

Mean values of dry weight content for individual experimental objects area presented in Figure 2. Comparison was made using matrix $\mathbf{C}_{3}$ and considering hypothesis $\mathrm{H}_{03}$ (7). The value of the Lawley-Hotelling statistics was at a level of 23.8, and the empirical significance level was lower than 0.05 . The second, third and fourth harvests were characterised by higher dry weight content than the first and fifth. The lower level of biomass production in the last series could be connected with pot-binding, hence for the proper determination of the effect of ozone on biomass production the second, third and fourth series should be taken into consideration (Heagle et al. 1995). Köllner and Krause (2002) observed no effect of ozone on the biomass ratio of white clover. However similar tendencies to those noted by Heagle et al. (1995) were found during the season - the lowest levels were recorded in the first and last series of both exposed clones of $T$. repens. 


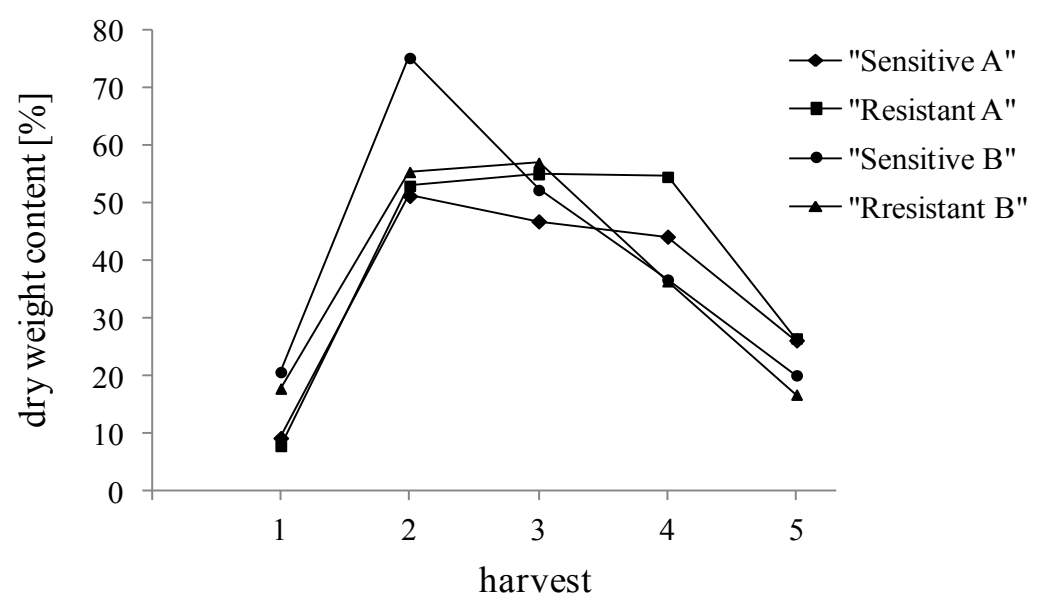

Figure 2. Profiles of dry weight content in ozone-sensitive and ozone-resistant clones of white clover for harvests A and B

The values of object effects are presented in Table 2. Based on these results it can be concluded that in the first and second harvest, lower levels of dry weight content [\%] of white clover were noted in series A than in series B (excluding the results obtained for the resistant clone in series B). Values of dry weight content in the fourth and fifth harvest were higher in series A than in series B. The third harvest was characterised by low variation of dry weight content.

Table 2. Values of experimental effect in terms of dry weight content [\%] of white clover

\begin{tabular}{ccccccc}
\hline \multirow{2}{*}{ Experimental objects } & \multicolumn{9}{c}{ Harvest } & Test statistics \\
\cline { 2 - 6 } & 1 & 2 & 3 & 4 & 5 & $T_{0 t}^{2}$ \\
\hline "Sensitive A" & $-4.66^{* *}$ & $-7.47 * *$ & $-5.95 *$ & 1.20 & $3.79 *$ & $16.81 * *$ \\
"Resistant A" & $-6.04 * *$ & $-5.71 *$ & 2.24 & $11.59 * *$ & $4.13 *$ & $27.85 * *$ \\
"Sensitive B" & $6.79 * *$ & $16.49 * *$ & -0.50 & $-6.25 *$ & -2.28 & $27.90 * *$ \\
"Resistant B" & $3.92 * *$ & -3.30 & 4.22 & $-6.54 *$ & $-5.64 * *$ & $24.41 * *$ \\
\hline
\end{tabular}

* significance level $\alpha=0.05 ; * *$ significance level $\alpha=0.01$

Values of object effects after transformation to Euclidean space are presented in Figure 3. It is possible to observe that the main direction of variation is connected with a division into experimental terms for series A and B. Points representing sensitive and resistant clones for series $\mathrm{A}$ are located on one side of 
the horizontal axis (characterised by high values for the fourth and fifth harvest and low for the first and the second).

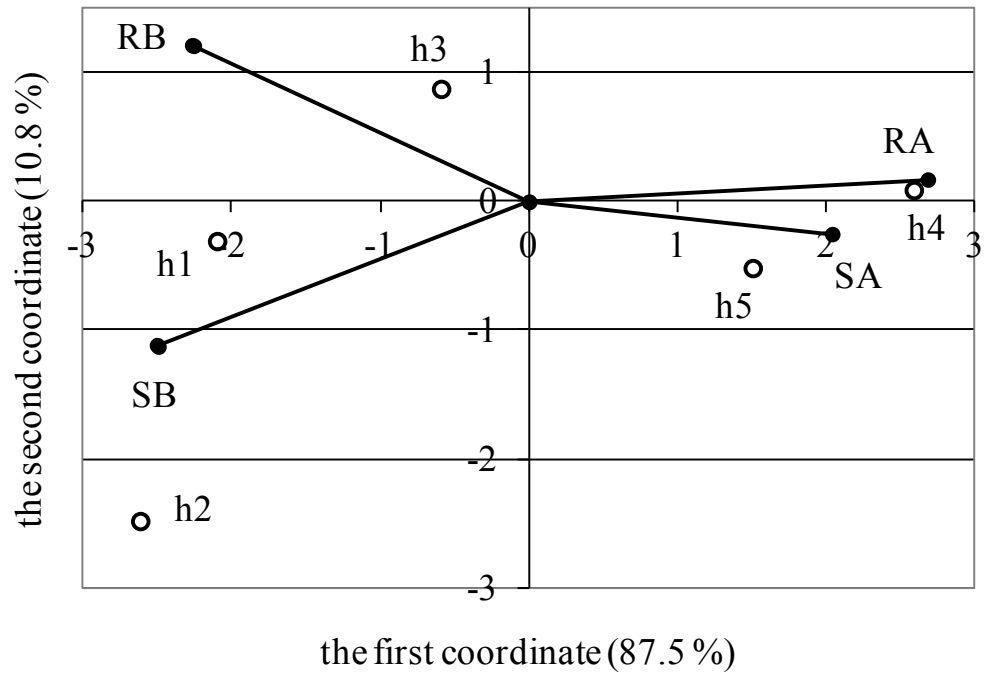

Figure 3. Canonical variate analysis of dry weight content of sensitive ("S") and resistant ("R") white clover clones in series A and B

The results of testing of the hypothesis of the parallelism of profiles of the sensitive clone in series $\mathrm{A}$ and $\mathrm{B}$ are presented in Table 3. Note that the sensitive clone in series A had lower dry weight content than in series B for harvests 1 and 2, but higher content in the case of harvest 5 . Furthermore, it is noted that the resistant clone in series A had higher dry weight content than in series B for harvests 4 and 5, and lower in the harvest 1 . Hence it is concluded that the existence of differences in dry weight content between sets confirms the validity of both series during the growing season.

The results of comparison for summarized values across all harvests for certain objects are presented in Table 4 . The mean values of dry weight content for the sensitive clone in series A were lower than in series B, while for the resistant clone the opposite tendency was observed. This means that different 
Table 3. Profile differences between dry weight content of white clover clones in certain harvests

\begin{tabular}{|c|c|c|c|c|c|c|}
\hline \multirow{3}{*}{ Difference } & & & Harvest & & & \multirow{3}{*}{ Test statistics } \\
\hline & 1 & 2 & 3 & 4 & 5 & \\
\hline & \multicolumn{5}{|c|}{ Dry weight content [\%] } & \\
\hline "Sensitive A" - "Sensitive B" & $-11.5 * *$ & $-24.0 * *$ & -5.45 & 7.45 & $6.07 *$ & $30.40^{* *}$ \\
\hline "Resistant A" - "Resistant B" & $-9.96 * *$ & -2.41 & -1.98 & $18.1 * *$ & $9.76^{* *}$ & $36.10^{* *}$ \\
\hline "Sensitive A" - "Resistant A" & 1.38 & -1.76 & -8.19 & -10.4 & -0.33 & 2.00 \\
\hline "Sensitive B" - "Resistant B" & $2.87 * *$ & $19.8 * *$ & -4.72 & 0.29 & 3.35 & $7.74 * *$ \\
\hline
\end{tabular}

times of harvest had a significant effect on dry weight content for certain white clover clones. Moreover, comparison of dry weight content between clones in certain harvests revealed that in series A the summarized values of dry weight content of the sensitive clone were lower than for the resistant one, while for series B they were higher. It seems to be very important to retain the overlapping series A and B, due to the lack of leaves after harvesting. White clover is one of the most important pasture species, hence it is important to evaluate the potential for survival of this species. Previous investigations revealed a decline in white clover fraction in response to long-term ozone treatment with low peak concentration (Nussbaum et al. 1995). Biomass production of NC-R was higher than NC-S, except for the harvest in one series. The yield increased in the first three series (Postiglione et al. 2000). Fumagilli et al. (2003) found a $60 \%$ decrease in the biomass production of a sensitive clone in comparison with a resistant one. This was not so clearly marked in our experiment, which may be connected with the relatively low ozone concentration during the growing season.

Table 4. Profile differences between mean values for all harvests of dry weight content of white clover clones

\begin{tabular}{lc}
\hline Difference & $\mathbf{c}_{t}^{\prime} \hat{\boldsymbol{\gamma}} \mathbf{1}_{H}$ \\
\hline "Sensitive A" - "Sensitive B" & -5.47 \\
"Resistant A" - "Resistant B" & 2.71 \\
"Sensitive A" - "Resistant A" & -3.86 \\
"Sensitive B" - "Resistant B" & 4.32 \\
"Sensitive" - "Resistant" & 0.23 \\
"A" - "B" & -1.38 \\
\hline
\end{tabular}




\section{Conclusions}

The dry weight of white clover exposed to ambient air ozone varied between harvests and clones. The experiment confirmed the necessity of supplementary exposure series to avoid a period without leaves. Moreover it is difficult to identify the directions of changes in the dry weight content of the clones, due to the high variability of the results. However, profile analysis and graphical presentation using canonical variate analysis was very useful in evaluating the changes of the examined parameters. It seems that dry matter content is not a good indicator of ozone effect on white clover in place of visible leaf injuries.

\section{REFERENCES}

Anderson T.W. (2003): An Introduction to Multivariate Statistical Analysis. Third Edition, John Wiley and Sons, New York.

Borowiak K., Kayzer D., Budka A., Zbierska J. (2011): Study of changes in the degree of tobacco leaf injury caused by tropospheric ozone. Biometrical Letters 48(1): 55-66.

Borowiak K., Kayzer D., Budka A., Zbierska J., Drzewiecka K., Bandurska H., Goliński P. (2012): Cumulative tropospheric ozone effect on visible tobacco leaf injury. Fresenius Environmental Bulletin 21(2a): 509-517.

Budka A., Borowiak K., Zbierska J., Kayzer D., Krzesinski W. (2011): Investigations of multidimensional linear model for comparison of tropospheric ozone-caused tobacco leaf injury degree between rural and urban exposure sites. Fresenius Environmental Bulletin 20(4): 969-975.

Caliński T., Świetlicka-Grala J., Grala B. (1975): Analiza doświadczeń z roślinami wieloletnimi i wielopokosowymi [Analysis of experiments with perennial and multi-cut plants]. Biuletyn Oceny Odmian 1(6): 117-138.

Elagöz V., Manning W.J. (2005): Responses of sensitive and tolerant bush beans (Phaseolus vulgaris L.) to ozone in open-top chambers are influenced by phenotypic differences, morphological characteristics, and the chamber environment. Environmental Pollution 136: 371-383.

Feng Z.Z., Kobayashi K., and Ainsworth E.A. (2008): Impact of elevated ozone concentration on growth, physiology and yield of wheat (Triticum aestivum L.): a meta-analysis. Global Change Biology 14: 2696-2708.

Fumagilli I., Mignanego L., Mills G. (2003): Ozone biomonitoring with clover clones: yield loss and carryover effect under high ambient ozone levels in northern Italy. Agriculture, Ecosystems and Environment 95: 119-128.

Hassan I.A., Tewfik I. (2006): $\mathrm{CO}_{2}$ photoassimilation, chlorophyll fluorescence, lipid peroxidation and yield in cotton (Gossypium hirsutum L. cv Giza 65) in response to 
$\mathrm{O}_{3}$. World Review of Science, Technology and Sustainable Development 3(1): 70-78.

Heagle A.S., Miller J.E., Chevone B.I., Dreschel T.W., Manning W.J., McCool P.M., Morrison C.L., Neely G.E., Rebbeck J. (1995): Response of a white clover indicator system to tropospheric ozone at eight locations in the United States. Water, Air and Soil Pollution 85: 1373-1378.

ICP Vegetation (2003): Experimental Protocol for the 2003 Season. International Cooperation Programme on effects of air pollution on natural vegetation and crops. Working group on effects. Centre of ecology and Hydrology. Bangor. Great Britain.

Kayzer D., Borowiak K., Budka A., Zbierska J. (2009): Study of interaction in bioindication research on tobacco plant injuries caused by ground level ozone. Environmetrics 20: 666-675.

Klumpp A., Ansel W., Klumpp G., Calatayud V., Garrec J.P., He S., Peñuelas J. Ribas À., Ro-Poulsen H., Rasmussen S., Sanz M.J., Vergne P. (2006): Ozone pollution and ozone biomonitoring in European cities. Part I: Ozone concentrations and cumulative exposure indices at urban and suburban sites - Atmos. Environ. 40: 7963-7974.

Köllner B., Krause G.H.M. (2002): Assessment of the response of the NC-S/NC-R clover clone system to ambient ozone levels at the Ruhr Valley. Water, Air and Soil Pollution 137: 63-79.

Lejeune M., Caliński T. (2000): Canonical analysis applied to multivariate analysis of variance. Journal of Multivariate Analysis 72: 100-119.

McKeon J.J. (1974): F approximations to the distribution of Hotelling's $T_{0}^{2}$. Biometrika 61: 381-383.

Morrison D.F. (1967): Multivariate Statistical Methods. New York.

Nussbaum S., Geissmann M., Fuhrer J. (1995): Ozone-exposure-response relationships for mixture of perennial ryegrass and white clover depend on ozone exposure patterns. Atmospheric Environment 29: 989-995.

Postiglione L., Fagnano M., Merola G. (2000): Response to ambient ozone of two white clover (Trifolium repens L. cv. "Regal") clones, one resistant and one sensitive, grown in a Mediterranean environment. Environmental Pollution 109: 525-531.

Rai R., Agrawal M., Agrawal S.B. (2007): Assessment of yield losses in tropical wheat using open top chambers. Atmospheric Environment 41: 9543-9554.

Seber G.A.F. (1980): The Linear Hypothesis: A General Theory. Charles Griffin. London.

Seber G.A.F. (1984): Multivariate Observations. Wiley. New York.

Shi G., Yang L., Wang Y., Kobayashi K., Zhu J., Tang H., Pan S., Chen T., Liu G., Wang Y. (2009): Impact of elevated ozone concentration on yield of four Chinese rice cultivars under fully open-air field conditions. Agriculture, Ecosystems and Environment 131: 178-184.

Van Dingenen R., Dentener F.J., Frank R., Maurten C.K., Emberson L., Cofala J. (2009): The global impact of ozone on agricultural crop yield under current and future air quality legislation. Atmospheric Environment 43: 604-618.

Vandermeiren K., Black C., Pleijel H., De Temmerman L. (2005): Impact of rising tropospheric ozone on potato: effects on photosynthesis, growth, productivity and yield quality. Plant, Cell and Environment 28: 982-996. 